\title{
Nonlinear One - Link Flexible Manipulator State Estimation Using the Extended Kalman Filter
}

\author{
Mohammed Bakhti and Badr Bououlid Idrissi \\ Moulay Ismaïl University, Ecole Nationale Supérieure d'Arts et Métiers, BP 4024, \\ Marjane II, Beni Hamed, 50000, Meknès, Morocco \\ mdbakhti@yahoo.fr,badr.bououlid@gmail.com
}

\begin{abstract}
Flexible manipulators active vibration control has been subject to a large number of research papers due to its high potential for industrial applications. However, this approach generally requires the feedback of state variables that are not always available via direct measurements. The present study proposes a state estimation scheme for a nonlinear one - link flexible manipulator based on the Extended Kalman Filter. First, the flexible manipulator, seen as an Euler-Bernoulli cantilever beam, is modeled using The Hamilton's principle, the elastic movement is approximated using the assumed modes method and the system non linear equations are introduced and converted into the matrix form. Then, the observability of the system is proven and the Extended Kalman Filter scheme is detailed. This article compares, via the numerical simulation results included, the efficiency of the state estimate, reflected by the estimation error and the time required by the filter to converge, relatively to the assumptions on the available measurements.
\end{abstract}

Keywords: Non linear State Estimation, one - link flexible manipulator, System observability, Extended Kalman Filter

\section{Introduction}

Having potential advantages of greater payload to manipulator weight ratio, lower energy consumption and faster response, the flexible manipulators are increasingly utilized in many modern applications such as aircraft, and space structures. However, their modeling approaches and their control/observation systems must consider both the rigid body and the flexible degrees of freedom [1].

One of the common used approaches, for modeling the one-link flexible manipulator, which is essential for the understanding of general multiple-link flexible manipulators, rely on the Hamilton's principle or the Lagrange equations of movement. Many research papers [2 - 7] have used the Euler - Bernoulli beam theory to derive the deformation model, while the elastic degree of freedom is approximated using either the assumed mode method or the finite element method.

Previous nonlinear observer formulations for the flexible manipulator have been investigated. An extended state observer was proposed for the trajectory tracking control of a flexible-joint robotic system in [8]. A nonlinear high gain observer has been developed to estimate the elastic degrees of freedom and their time derivatives in [9], and the Extended Kalman Filter has been used in [9] to give an estimate of the environmental forces. The sliding mode theory has been investigated in [11] to design both a controller and an observer for the tip positioning problem. Distributed observers have been presented in $[12,13]$ to estimate infinite dimensional states requiring only the boundary values measured by sensors.

The principal contribution of this paper is to formulate the Extended Kalman filter, based on the nonlinear model of the one-link flexible manipulator, to give an estimate of its state variables. The analyzed motion of the flexible one-link manipulator consists of 
the rigid body motion, defined by the joint angle, and the vibration motion, defined by the first two modal coordinates. The flexible arm deformation is described using the Euler-Bernoulli theory and the system equations are derived using Hamilton's principle. Two sets of equations, associated with the rigid body and the elastic degrees of freedom and considering the flexible link structural damping, are detailed in section 2. Section 3 deals with the Extended Kalman Filter formulation to give an estimate of the system states based on the available noisy measurements. First, only the joint angle will be used as an available output and the observability of the system will be proven using the observability matrix. Then the tip vibration derivative will also be considered as an output to better the estimate results. Two sources of zero-mean Gaussian white noise will be affecting the control torque and the available measurements. In section 4, simulation will be conducted using SIMULINK models and results are discussed for both cases. Section 5 concludes the study.

\section{Dynamic Model of the Flexible Manipulator}

In this section, the mathematical model of the flexible one-link manipulator, used for simulation and observer design, will be detailed. The model is developed for the case of a manipulator with one flexible link, assumed to be an Euler-Bernoulli clamped-free beam and constrained to act on a horizontal plane. It is rigidly connected, via a rigid hub, to the shaft of an electric servomotor.

Two reference systems are defined:

1. An inertial system: $(X, Y, Z)$ with its origin at the centre of the hub, its $Z$-axis aligned with the electric servomotor shaft, and the $X$-axis aligned with the home position of the flexible manipulator.

2. A rotating system: $(x, y, Z)$, as local coordinate system, attached to the hub and its $x$-axis tangent to the link at the shaft.

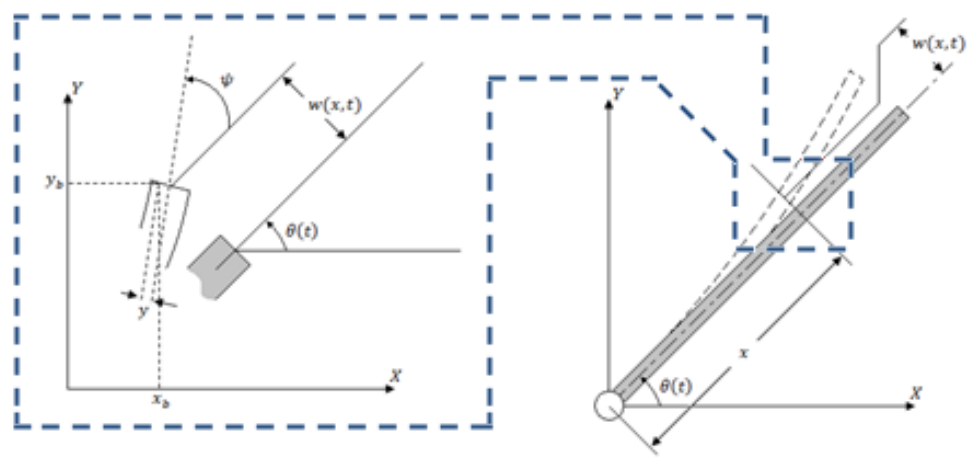

Figure 1. Flexible Manipulator Geometry and Coordinates

The manipulator motion is described by the rotation angle $\theta(t)$, the flexible displacement $w(x, t)$ and the transverse section rotation $\psi(x, t)$. Given the EulerBernoulli beam assumption, any transverse section of the beam remains a plane and normal to the longitudinal axis of the beam after deformation [14]. In this case, the shear deformation of the beam is neglected, and:

$$
\psi(x, t)=\frac{\partial w(x, t)}{\partial x}
$$

A more accurate assumption states that any transverse section of the beam remains plane, but it may not be perpendicular to the longitudinal axis. If this is the case, the shear deformation of the beam is not neglected, and, as a result, $\psi$ is independent of $w$. This is the well-known Timoshenko beam assumption [15].

The system equations are derived using Hamilton's principle [16]: 


$$
\int_{t_{0}}^{t_{f}}(\delta T-\delta P+\delta W) d t=0
$$

Where $\mathrm{T}$ and $\mathrm{P}$ are, respectively, the kinetic and potential energies of the system, and $\mathrm{W}$ is the work done by external forces.

The position of a point on the beam, having $(x, y)$ coordinates before deformation, is described in the inertial system by:

$$
\left\{\begin{array}{l}
x_{b}=x \cos (\theta(t))-w(x, t) \sin (\theta(t))-y \sin (\theta(t)+\psi(x, t)) \\
y_{b}=x \sin (\theta(t))+w(x, t) \cos (\theta(t))-y \cos (\theta(t)+\psi(x, t))
\end{array}\right.
$$

The kinetic energy of the beam is given by [17]:

$$
T_{b}=\frac{1}{2} \int_{0}^{L} \rho A\left[(x \dot{\theta}+\dot{w})^{2}+(w \dot{\theta})^{2}+\frac{I}{A}(\dot{\theta}+\dot{\psi})^{2}\right] d x
$$

Where $\rho, A$ and $I$ are respectively the mass density of the beam, its cross section area and its moment of inertia.

Including the motor and hub inertia $I_{H}$, the kinetic energy of the system is:

$$
T=\frac{1}{2} I_{H} \dot{\theta}^{2}+\frac{1}{2} \int_{0}^{L} \rho A\left[(x \dot{\theta}+\dot{w})^{2}+(w \dot{\theta})^{2}+\frac{I}{A}(\dot{\theta}+\dot{\psi})^{2}\right] d x
$$

The potential energy of the flexible manipulator is given by [16]:

$$
P=\frac{1}{2} \int_{0}^{L} E I\left(\frac{\partial^{2} w(x, t)}{\partial x^{2}}\right)^{2} d x
$$

Where $\mathrm{E}$ is the Young's modulus of the beam, and $E I$ its bending rigidity.

Let $\tau$ be the torque applied at the hub, the work done by external forces to the flexible manipulator is:

$\delta W=\tau \delta \theta$

The assumed modes method is used to approximate $w(x, t)$. The relative motion of the link with respect to the rotating reference system will be written in terms of the modal coordinates $q_{i}(t)$ and the clamped-free beam's mode shapes $\varphi_{i}(x)$ :

$$
w(x, t)=\sum_{i=1}^{N} q_{i}(t) \varphi_{i}(x)
$$

In our study, simulation of the system equation is conducted based on the first two elastic modes $(N=2)$. However, during the observer design, the second elastic mode is ignored.

The Hamilton's principle results on the following equations governing the rigid motion of the beam (function of $\theta(t)$ ), and the flexible one (function of $q_{1}(t)$ and $q_{2}(t)$ ).

$$
\begin{aligned}
& {\left[E I \int_{0}^{L} \frac{\partial^{4} \varphi_{1}(x)}{\partial x^{4}} d x\right] q_{1}+\left[E I \int_{0}^{L} \frac{\partial^{4} \varphi_{2}(x)}{\partial x^{4}} d x\right] q_{2}+\rho A \frac{L^{2}}{2} \ddot{\theta}} \\
& +\left[\left(\rho A \int_{0}^{L} \varphi_{1}(x) d x\right)-\left(\rho I \int_{0}^{L} \frac{\partial^{2} \varphi_{1}(x)}{\partial x^{2}} d x\right)\right] \ddot{q}_{1} \\
& +\left[\left(\rho A \int_{0}^{L} \varphi_{2}(x) d x\right)-\left(\rho I \int_{0}^{L} \frac{\partial^{2} \varphi_{2}(x)}{\partial x^{2}} d x\right)\right] \ddot{q}_{2} \\
& -\left[\left(\rho A \int_{0}^{L} \varphi_{1}(x) d x\right) q_{1}-\left(\rho A \int_{0}^{L} \varphi_{2}(x) d x\right) q_{2}\right] \dot{\theta}^{2} \\
& \left.+I_{H}+\rho A \frac{L^{3}}{3}+\rho I L\right) \ddot{\theta} \\
& +\left[\left(\rho A \int_{0}^{L} x \varphi_{1}(x) d x\right)+\left(\rho I \int_{0}^{L} \frac{\partial \varphi_{1}(x)}{\partial x} d x\right)\right] \ddot{q}_{1} \\
& +\left[\left(\rho A \int_{0}^{L} x \varphi_{2}(x) d x\right)+\left(\rho I \int_{0}^{L} \frac{\partial \varphi_{2}(x)}{\partial x} d x\right)\right] \ddot{q}_{2} \\
& +\left[\left(\rho A \int_{0}^{L} \varphi_{1}^{2}(x) d x\right) q_{1}^{2}++\left(\rho A \int_{0}^{L} \varphi_{2}^{2}(x) d x\right) q_{2}^{2}\right] \ddot{\theta}
\end{aligned}
$$




$$
\begin{aligned}
& +2\left[\left(\rho A \int_{0}^{L} \varphi_{1}^{2}(x) d x\right) q_{1} \dot{q}_{1}+2\left(\rho A \int_{0}^{L} \varphi_{2}{ }^{2}(x) d x\right) q_{2} \dot{q}_{2}\right] \dot{\theta} \\
& \quad=\tau
\end{aligned}
$$

These equations can be rearranged so as to obtain the following general form:

$$
M(q) \ddot{q}+h(q, \dot{q})+K q=u(t)
$$

Where $q$ is the vector of generalized coordinate including the joint angle and the elastic modal coordinates.

$$
q=\left[\begin{array}{c}
\theta(t) \\
q_{1}(t) \\
q_{2}(t)
\end{array}\right]
$$

Matrices $M(q)$ and $K$ are respectively the mass and the stiffness ones, and the vector $h(q, \dot{q})$ regroups the non linear centrifugal and Coriolis terms. The vector of external forces is given by:

$$
u(t)=\left[\begin{array}{l}
\tau \\
0 \\
0
\end{array}\right]
$$

In addition, the motor viscous friction coefficient $\alpha_{m}$ and the link structural damping can form a modal damping matrix $H_{d}$ as [18]:

$$
H_{d}=\left[\begin{array}{ccc}
\alpha_{m} & 0 & 0 \\
0 & 2 \xi_{1} m_{22} \omega_{1} & 0 \\
0 & 0 & 2 \xi_{2} m_{33} \omega_{2}
\end{array}\right]
$$

Where $\omega_{1}, \omega_{2}$ are the first two elastic modes natural frequencies, and $\xi_{1}, \xi_{2}$ their respective modal damping coefficients. Coefficients $m_{22}$ and $m_{33}$ are the corresponding elements of the mass matrix $M(q)$.

The mathematical model of the system considering the modal damping matrix is given by:

$$
M(q) \ddot{q}+H_{d} \dot{q}+h(q, \dot{q})+K q=u(t)
$$

The matrices and their numerical values used for simulation are detailed in appendix A.

Finally, the model that will be used for simulation and observer design is given by:

$\dot{x}=f(x, u), \quad x(0)=x_{0}$

Where:

$$
\dot{x}=\left[\begin{array}{llllll}
\theta & q_{1} & q_{2} & \dot{\theta} & \dot{q}_{1} & \dot{q}_{2}
\end{array}\right]^{T}
$$

$$
\text { And: }
$$

$f=\left[\begin{array}{c}\dot{\theta} \\ \dot{q}_{1} \\ \dot{q}_{2} \\ -M(q)^{-1} H_{d} \dot{q}-M(q)^{-1} h(q, \dot{q})-M(q)^{-1} K q+M(q)^{-1} u(t)\end{array}\right]$

\section{State Estimation and Observer Design}

This section deals with the observer design. The observability of the system is discussed regarding the measurable inputs and outputs, and the Extended Kalman Filter (EKF) equations are detailed.

\subsection{State Estimation Problem}

The EKF is used to estimate the angular position of the hub, the first modal coordinate and their respective derivatives. The estimation will be based on the noisy available measurements, and the state estimate is given by:

$$
\hat{x}=\left[\begin{array}{llll}
\hat{\theta} & \hat{q}_{1} & \dot{\hat{\theta}} & \dot{\hat{q}}_{1}
\end{array}\right]^{T}
$$

The flexible manipulator model, as formulated in section (II), is given by: 


$$
\left\{\begin{array}{c}
\dot{x}=f(x, u), \quad x(0)=x_{0} \\
y=h(x)
\end{array}\right.
$$

The measurement equation $h($.$) will depend on the available measurements.$

It is assumed that the system is locally observable if the observability matrix:

$$
Q(x, u)=\left[\begin{array}{c}
N_{f}^{0} \\
N_{f}^{1} \\
\vdots \\
N_{f}^{n-1}
\end{array}\right] \frac{\partial h}{\partial x}
$$

has full rank in the considered domains of $x$ and $u$ [19].

The linear differential operator $N_{f}$, that is repeatedly applied to the row vector $\frac{\partial h}{\partial x}$, is defined by [19]:

$$
\begin{aligned}
& N_{f}\left(\frac{\partial h}{\partial x}\right)=\frac{\partial h}{\partial x} \frac{\partial f}{\partial x}+f^{T} \frac{\partial}{\partial x}\left(\frac{\partial h}{\partial x}\right)^{T}+\dot{\bar{u}}^{T} \frac{\partial}{\partial \bar{u}}\left(\frac{\partial h}{\partial x}\right)^{T} \\
& \text { With } \\
& N_{f}^{0}\left(\frac{\partial h}{\partial x}\right)=\frac{\partial h}{\partial x} \\
& \text { And } \\
& N_{f}^{1}\left(\frac{\partial h}{\partial x}\right)=N_{f}^{0}\left(N_{f}^{0}\left(\frac{\partial h}{\partial x}\right)\right)
\end{aligned}
$$

The vector $\overline{\mathrm{u}}$ is given by:

$$
\bar{u}=\left[\begin{array}{llll}
u & \dot{u} & \ldots & u^{n-1}
\end{array}\right]^{T}
$$

Assuming that only the angular position of the hub $\theta(t)$ is measured, the local observability of the system can be proven.

Using equation (21) to construct matrix $Q(x, u)$ yields:

$$
Q(x, u)=\left[\begin{array}{cccc}
1 & 0 & 0 & 0 \\
0 & 0 & 1 & 0 \\
0 & P_{2}(q) & P_{3}(q) & P_{4}(q) \\
0 & Q_{2}(q) & Q_{3}(q) & Q_{4}(q)
\end{array}\right]
$$

Where the $P_{i}$ and $Q_{i}$ polynomials, described in appendix $\mathrm{B}$, are in terms of state variables, and they are nonzero. Thus the independence of the rows in matrix $Q(x, u)$ is ensured, which guarantees the local observability of the system.

\subsection{The Extended Kalman Filter}

The Extended Kalman Filter (EKF) is an extension of the Kalman Filter scheme to nonlinear systems. This method makes possible an accurate online estimation of states taking the system errors and measurement noises into account.

The state of the flexible manipulator is assumed to be a stochastic process and the design of the EKF is based on the following continuous-time, stochastic and non linear model of the system:

$$
\left\{\begin{array}{c}
\dot{x}=f(x, u, w) \\
y=h(x, v)
\end{array}\right.
$$

First, simulation will be conducted assuming that only the angular position of the hub is measured. Then the results are compared to the case where the modal coordinate derivative measurement is also available.

The system equation $f($.$) is a non linear vector function, and w(t)$ and $v(t)$ are the process and measurement noises respectively. The noises are assumed to be continuoustime, white, zero-mean, uncorrelated and have known covariance matrices $Q$ and $R$ respectively.

$$
\left\{\begin{array}{l}
E[(w(t))(w(\tau)]=Q \delta(t-\tau) \\
E[(v(t))(v(\tau)]=R \delta(t-\tau)
\end{array}\right.
$$


Where $E[$.$] and \delta($.$) denote, respectively, the expected value and the continuous-time$ impulse function.

The EKF scheme is based on the extended model of the given nonlinear system. Taylor series are used to linearize both the system and the measurement functions around a nominal trajectory as follows:

$$
\left\{\begin{array}{c}
\dot{x}=f\left(x_{0}, u_{0}, w_{0}\right)+\left.\frac{\partial f}{\partial x}\right|_{0}\left(x-x_{0}\right)+\left.\frac{\partial f}{\partial u}\right|_{0}\left(u-u_{0}\right)+\left.\frac{\partial f}{\partial w}\right|_{0}\left(w-w_{0}\right) \\
y=h\left(x_{0}, v_{0}\right)+\left.\frac{\partial h}{\partial x}\right|_{0}\left(x-x_{0}\right)+\left.\frac{\partial h}{\partial v}\right|_{0}\left(v-v_{0}\right)
\end{array}\right.
$$

Since the process noise and the measurement noise are assumed to be zero-mean signals, their nominal trajectories $w_{0}(t)$ and $v_{0}(t)$ are logically both equal to zero. It's also considered that the control signal $\mathrm{u}(\mathrm{t})$ is perfectly known which means that $u_{0}(t)=$ $u(t)$, and the state nominal trajectory in the EKF algorithm is the state estimate itself $x_{0}(t)=\hat{x}(t)$.

The EKF equations are given by [20]:

$$
\left\{\begin{array}{c}
\hat{x}(0)=E[x(0)] \\
P(0)=E\left[(x(0)-\hat{x}(0))(x(0)-\hat{x}(0))^{T}\right] \\
\dot{\hat{x}}=f\left(\hat{x}, u, w_{0}\right)+K\left(y-h\left(\hat{x}, w_{0}\right)\right) \\
K=P C^{T} \tilde{R}^{-1} \\
\dot{P}=A P+P A^{T}+\tilde{Q}-P C^{T} \tilde{R}^{-1} C P
\end{array}\right.
$$

Where $\mathrm{P}$ is the covariance of the estimation error, and the nominal noise values are given as $w_{0}=0$ and $v_{0}=0$. Matrices $A, C, \tilde{Q}, L, \tilde{R}$ and $M$ are defined as follows:

$$
\left\{\begin{array}{c}
A=\left.\frac{\partial f}{\partial x}\right|_{\hat{x}} \\
C=\left.\frac{\partial f}{\partial x}\right|_{\hat{x}} \\
\tilde{Q}=L Q L^{T} \\
L=\left.\frac{\partial f}{\partial w}\right|_{\hat{x}} \\
\tilde{R}=M R M^{T} \\
M=\left.\frac{\partial f}{\partial v}\right|_{\hat{x}}
\end{array}\right.
$$

\section{Simulation Results}

\begin{tabular}{|c|c|c|c|c|c|}
\hline \multirow{6}{*}{$\begin{array}{l}\text { The } \\
\text { beam }\end{array}$} & Mass density & $\rho=2700 \mathrm{Kg} \cdot \mathrm{m}^{-3}$ & \multirow{6}{*}{$\begin{array}{l}\text { The } \\
\text { DC } \\
\text { Motor }\end{array}$} & \multirow{3}{*}{$\begin{array}{l}\text { Motor and } \\
\text { hub inertia }\end{array}$} & \multirow{3}{*}{$I_{H}=0.08 \mathrm{Kg} \cdot \mathrm{m}^{2}$} \\
\hline & $\begin{array}{l}\text { Mass density per } \\
\text { unit length }\end{array}$ & $\rho A=0.15 \mathrm{Kg} \cdot \mathrm{m}^{-1}$ & & & \\
\hline & Beam length & $L=1 \mathrm{~m}$ & & & \\
\hline & Young's modulus & $E=6.910^{10} \mathrm{~Pa}$ & & \multirow{3}{*}{$\begin{array}{l}\text { Viscous } \\
\text { friction } \\
\text { coefficient }\end{array}$} & \multirow{3}{*}{$\alpha_{m}=0.95 \mathrm{Nm} \cdot r d^{-1} \cdot \mathrm{s}^{-1}$} \\
\hline & Flexural rigidity & $E I=1 N \cdot m^{2}$ & & & \\
\hline & $\begin{array}{c}\text { The quadratic } \\
\text { moment }\end{array}$ & $I=1.4510^{-9} \mathrm{~m}^{4}$ & & & \\
\hline
\end{tabular}

Two cases are assessed during the simulation. First, only the joint angle $\theta(t)$ is assumed to be an available measurement using a rotative high precision potentiometer. Discussion will be focused on the observability assessment and the ability of the filter to give an estimate of the system state $\hat{x}(t)$.

\section{Table 1. Numerical Parameters of the System}


The second simulation considers the tip vibration derivative $\dot{w}(L, t)$ also as an available measurement. This output can be easily integrated using an accelerometer bounded next to the tip of the beam. SIMULINK models were constructed in both cases.

The system has one input which is the mechanical torque $\tau(t)$, and table 1 . shows the beam and the DC motor parameters needed for the numeric simulation.

The process model is subject to two sources of zero-mean Gaussian white noise. The control torque $\tau(t)$ is corrupted with noise having a variance of $0.01^{2}$. The noises affecting the joint angle and the tip vibration derivative measurement are having variances of $0.01^{2}$ and $0.001^{2}$ respectively. Thus the variance matrices are summarized in Table 2.

\section{Table 2. Measurement Equation and Variance Matrices of Input and Measurements Noises}

\begin{tabular}{|ccc|}
\hline \multicolumn{3}{|c|}{ Case 1 } \\
\hline Input & $\tau(t)$ & $Q=\operatorname{Diag}_{4 \times 4}\left(0.01^{2}\right)$ \\
\hline Output & $y(t)=\theta(t)$ & $R=0.01^{2}$ \\
\hline \multicolumn{3}{|c|}{ Case 2 } \\
\hline Input & $\tau(t)$ & $Q=\operatorname{Diag}_{4 \times 4}\left(0.01^{2}\right)$ \\
\hline Outputs & $y(t)=\left[\begin{array}{c}\theta(t) \\
\dot{w}(L, t)\end{array}\right]$ & $R=\operatorname{Diag}_{2 \times 2}\left(0.01^{2}\right)$ \\
\hline
\end{tabular}

The observer design is based only on the first elastic mode to include the effect of the second one in the simulation. Initial state included in the truth model is supposed to be:

$x_{0}=\left[\begin{array}{llll}\theta_{0} & q_{10} & \dot{\theta}_{0} & \dot{q}_{10}\end{array}\right]^{T}=[0]_{4 \times 1}$

while the filtering scheme use initial state given by:

$\hat{x}_{0}=\left[\begin{array}{llll}\hat{\theta}_{0} & \hat{q}_{10} & \dot{\hat{\theta}}_{0} & \dot{\hat{q}}_{10}\end{array}\right]^{T}=\left[\begin{array}{llll}0 & 0.01 & 0.1 & 0\end{array}\right]^{T}$

The initial error covariance is assumed to be: $P(0)=I_{4 \times 4}$

Figure 2 to Figure 4 show respectively the control torque used for the simulation, the joint angle measurement and the tip vibration derivative measurement. The exact signals are fed to the truth model, while the noisy ones are fed to the EKF.

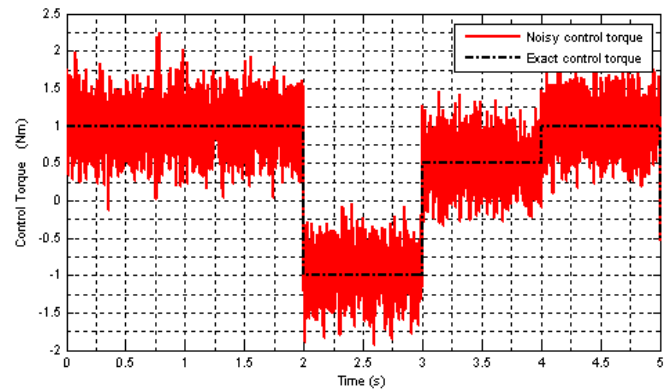

Figure 2. The Control Torque Used For the Simulation

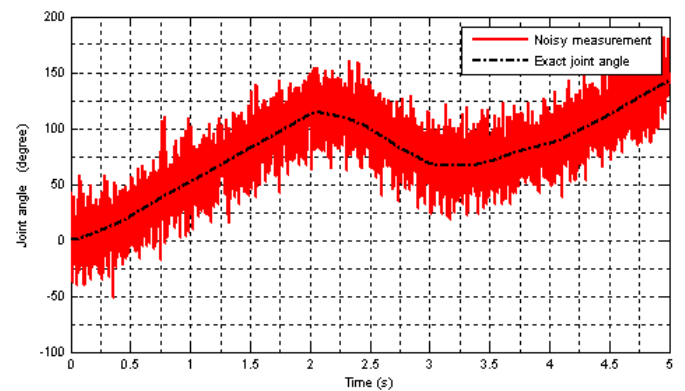

Figure 3. The Joint Angle Measurement 


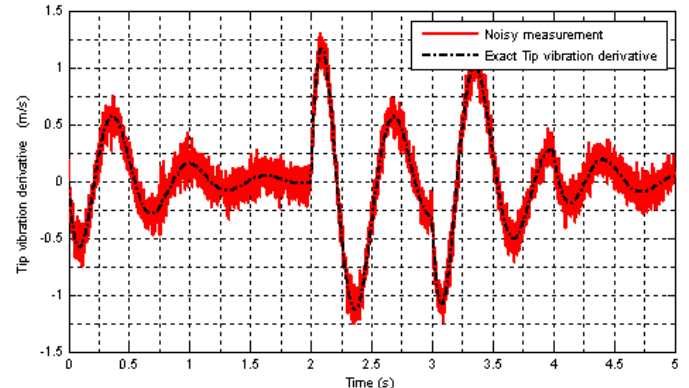

Figure 4. The Tip Vibration Derivative Measurement

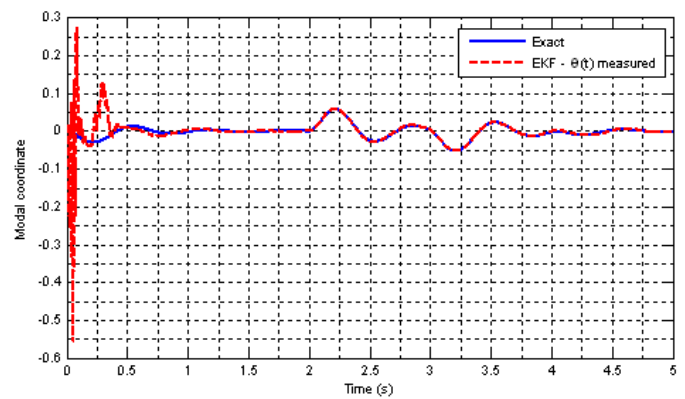

Figure 6. Estimation of the Modal Coordinate $q_{1}(t)$ Using $\theta(t)$ As Measurement

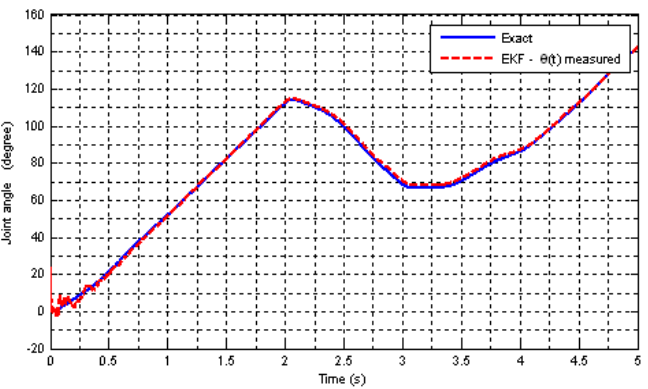

Figure 5. Estimation of the Joint Angle Using $\theta(t)$ As Measurement

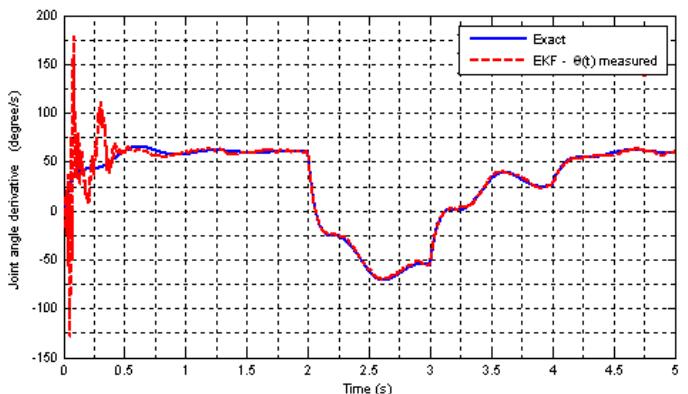

Figure 7. Estimation of the Joint Angle Derivative $\dot{\theta}(t)$ Using $\theta(t)$ As Measurement

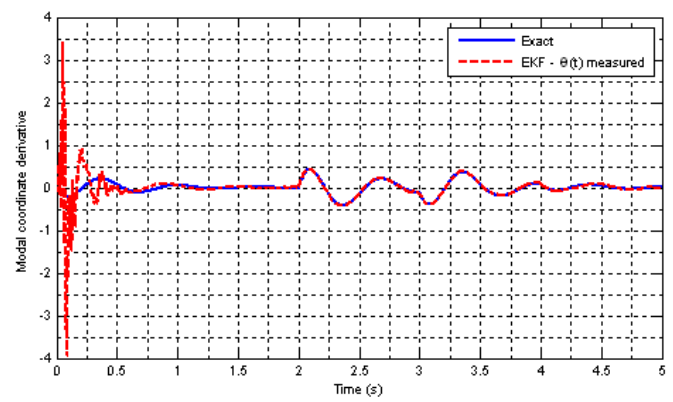

Figure 8. Estimation of the Modal Coordinate Derivative $\dot{\mathbf{q}}_{1}(\mathrm{t})$ Using $\theta(t)$ As Measurement

According to the results illustrated in Figure 5 to Figure 8, the EKF give a good estimate of the state. After a short transient time, the estimation error is small indicating that the system is observable using only the joint angle $\theta(t)$ as an available measurement.

Figure 9 to Figure 12 show that the estimation is better when the tip vibration derivative is supplied to the EKF. The error is smaller and the transient time is shorter. 


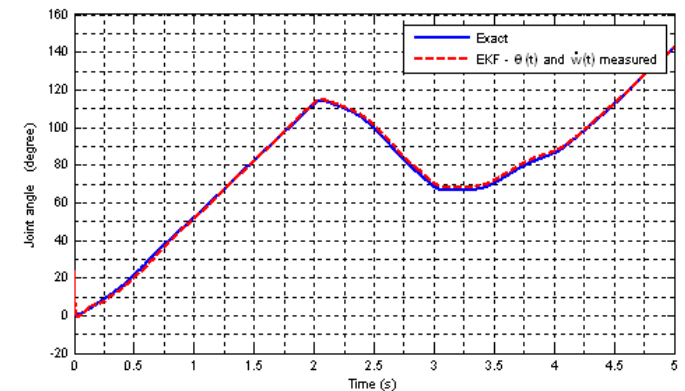

Figure 9. Estimation of the Joint Angle Using $\theta(t)$ and $\dot{w}(t)$ As Measurements

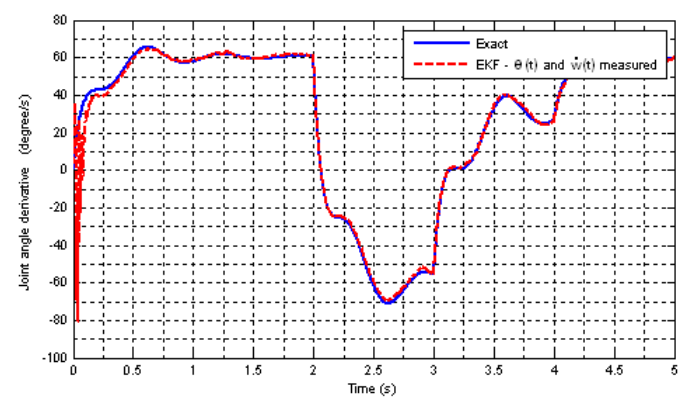

Figure 11. Estimation of the Joint Angle Derivative Using $\theta(t)$ and $\dot{w}(t)$ As Measurements

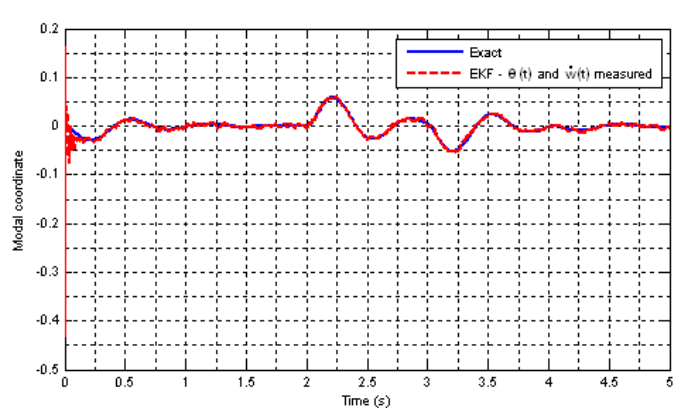

Figure 10. Estimation of the Modal Coordinate Using $\theta(t)$ and $\dot{w}(t)$ As Measurements

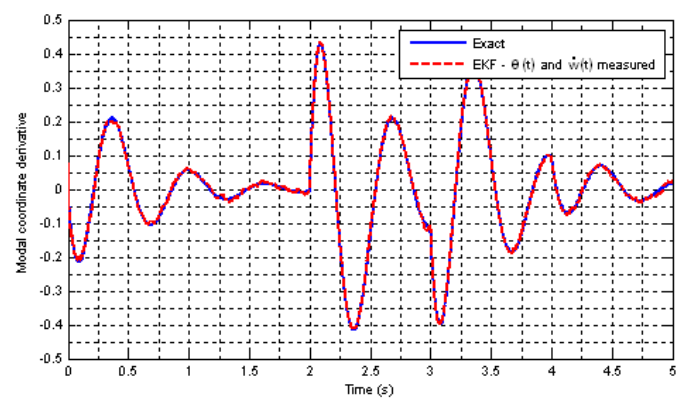

Figure 12. Estimation of the Modal Coordinate Derivative Using $\theta(t)$ and $\dot{w}(t)$ As Measurements

The state estimation errors, that represents the difference between the real values of $\theta(t), q(t), \dot{\theta}(t)$ and $\dot{q}(t)$ and their estimated values are shown in Figure 13 to Figure 16 As expected, the noisy measurement of the tip vibration derivative reduces widely both the estimation error and the required time for the EKF to converge.

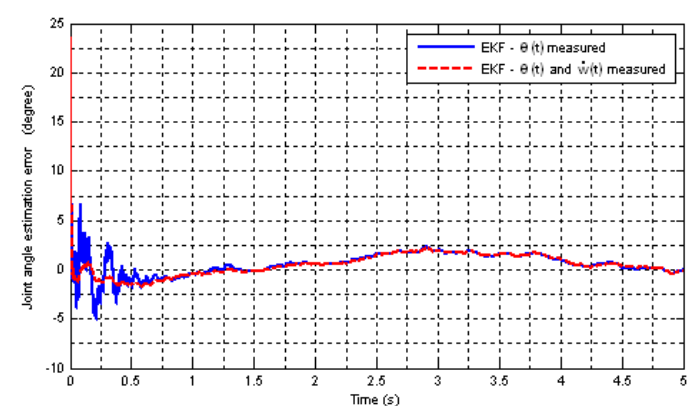

Figure 13. Estimation Error for the Joint Angle

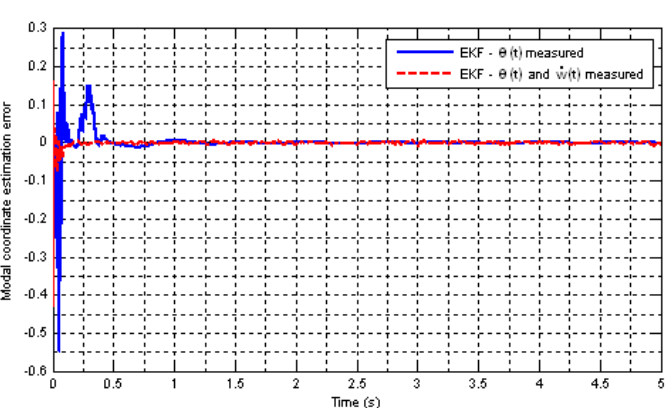

Figure 14. Estimation Error for the Modal Coordinate 


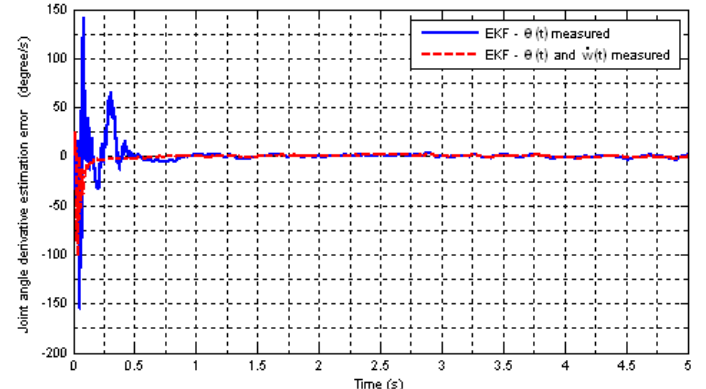

Figure 15. Estimation Error for
the Joint Angle Derivative

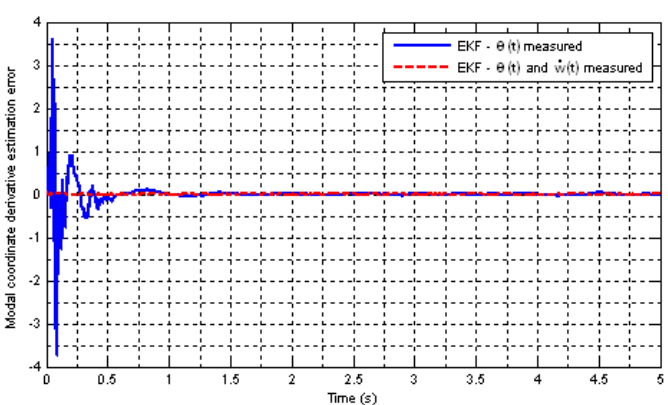

Figure 16. Estimation Error for the Modal Coordinate Derivative

\section{Conclusion}

This paper considers the problem of state estimation for a flexible one-link manipulator. Its primary contribution is the implementation of the EKF scheme based on the developed nonlinear model and restricted accessible noisy outputs.

The flexible manipulator was modeled using the Hamilton's principle and the assumed modes method considering the first two elastic modes. The state space representation obtained was used to conduct simulation and to discuss the observability of the system based either only on the joint angle measurement or on the tip vibration derivative as second available measurement.

Numerical simulations, conducted using SIMULINK models, demonstrate the effectiveness of the EKF to give a good estimate of the unmeasured states and the contribution of the second measurement to lower estimation error and required time for the EKF to converge.

\section{Appendix A. The Model Matrices and Their Numerical Values Used for Simulation}

The Mass Matrix

$$
\begin{aligned}
& M(q)== \\
& {\left[\begin{array}{ccc}
\left(I_{H}+\rho A \frac{L^{3}}{3}+\rho I L\right)+\rho A\left(\int_{0}^{L} \varphi_{1}(x)^{2} d x\right) q_{1}{ }^{2}+\left(\int_{0}^{L} \varphi_{2}(x)^{2} d x\right) q_{2}{ }^{2} & \rho A \int_{0}^{L} x \varphi_{1}(x) d x+\rho I \int_{0}^{L} \frac{d \varphi_{1}(x)}{d x} d x & \rho A \int_{0}^{L} x \varphi_{2}(x) d x+\rho I \int_{0}^{L} \frac{d \varphi_{2}(x)}{d x} d x \\
\rho A \int_{0}^{L} x \varphi_{1}(x) d x & \rho A \int_{0}^{L} \varphi_{1}(x)^{2} d x-\rho I \int_{0}^{L} \frac{d^{2} \varphi_{1}(x)}{d x^{2}} \varphi_{1}(x) d x & 0 \\
\rho A \int_{0}^{L} x \varphi_{2}(x) d x & 0 & \rho A \int_{0}^{L} \varphi_{2}(x)^{2} d x-\rho I \int_{0}^{L} \frac{d^{2} \varphi_{2}(x)}{d x^{2}} \varphi_{2}(x) d x
\end{array}\right]} \\
& =\left[\begin{array}{ccc}
0.13+0.2783 q_{1}{ }^{2}+0.1445 q_{2}{ }^{2} & 0.1162 & 0.0134 \\
0.1162 & 0.2783 & 0 \\
0.0134 & 0 & 0.1445
\end{array}\right]
\end{aligned}
$$

The vector of non linear centrifugal and Coriolis terms

$$
h(q, \dot{q})=\left[\begin{array}{c}
2 \rho A\left(\int_{0}^{L} \varphi_{1}(x)^{2} d x\right) q_{1} \dot{q}_{1} \dot{\theta}+2 \rho A\left(\int_{0}^{L} \varphi_{2}(x)^{2} d x\right) q_{2} \dot{q}_{2} \dot{\theta} \\
-\rho A\left(\int_{0}^{L} \varphi_{1}(x)^{2} d x\right) q_{1} \dot{\theta}^{2} \\
-\rho A\left(\int_{0}^{L} \varphi_{2}(x)^{2} d x\right) q_{2} \dot{\theta}^{2}
\end{array}\right]=\left[\begin{array}{c}
0.5566 q_{1} \dot{1}_{1} \dot{\theta}+0.2891 q_{2} \dot{q}_{2} \dot{\theta} \\
-0.2783 q_{1} \dot{\theta}^{2} \\
-0.1445 q_{2} \dot{\theta}^{2}
\end{array}\right]
$$

The stiffness matrix

$$
K(q)=\left[\begin{array}{ccc}
0 & 0 & 0 \\
0 & E I \int_{0}^{L}\left(\frac{d^{2} \varphi_{1}(x)}{d x^{2}}\right)^{2} d x & 0 \\
0 & 0 & E I \int_{0}^{L}\left(\frac{d^{2} \varphi_{2}(x)}{d x^{2}}\right)^{2} d x
\end{array}\right]=\left[\begin{array}{ccc}
0 & 0 & 0 \\
0 & 22.94 & 0 \\
0 & 0 & 467.9280
\end{array}\right]
$$




\section{Appendix B. The Observability Matrix Polynomials}

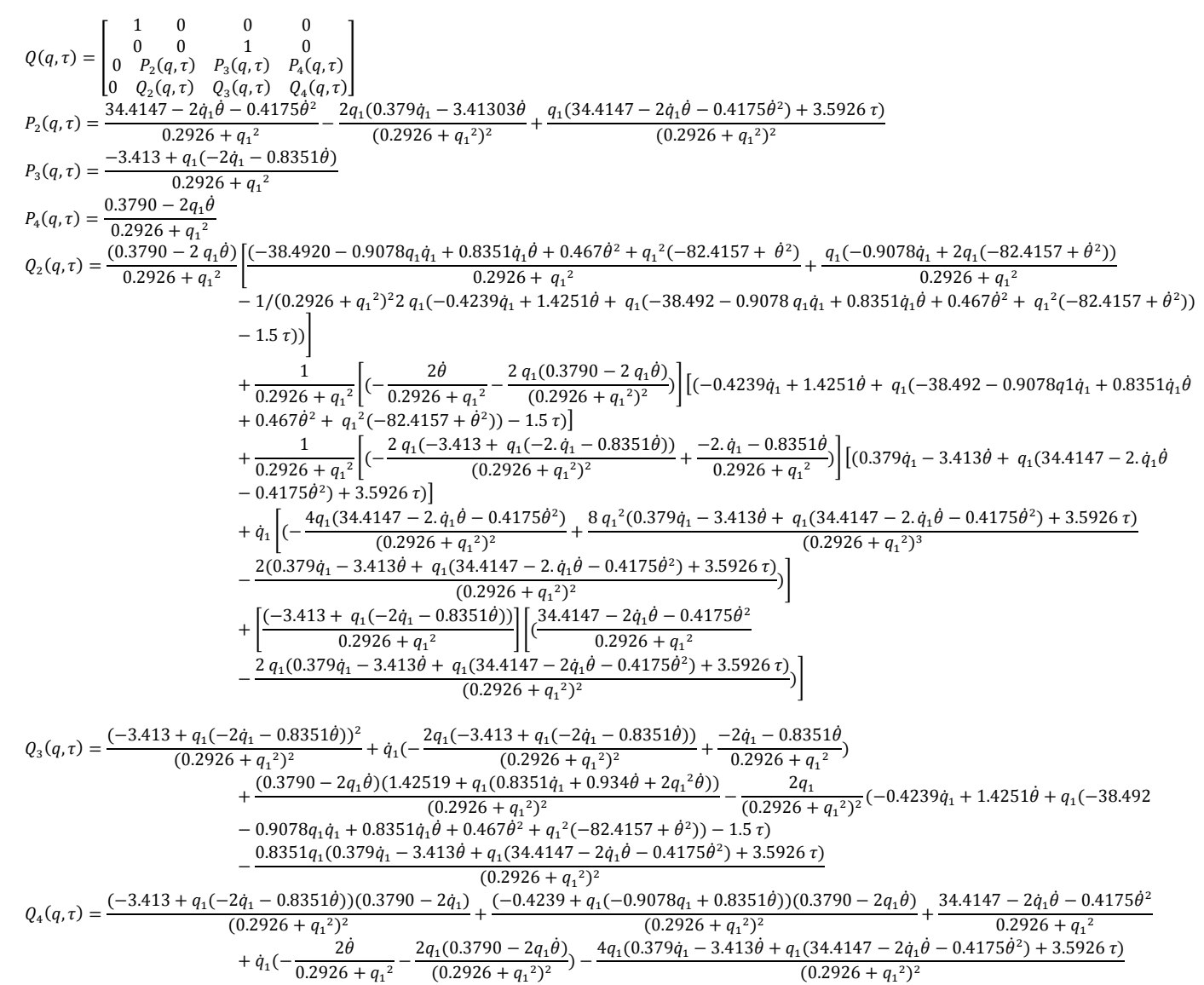

\section{References}

[1] S.K. Dwivedy and P. Eberhard, "Dynamic analysis of flexible manipulators, a literature review", Mechanism and Machine Theory, vol. 41, no. 7, (2006), pp. 749-777.

[2] F. Bellezza, L. Lanari and G. Ulivi, "Exact modeling of the flexible slewing link", Proceedings of the IEEE International Conference on Robotics and Automation, Cincinnati, USA, (1990), pp. 734-739.

[3] R.H. Cannon Jr. and E. Schmitz, "Precise control of flexible manipulators", Robotics Research: First International Symposium, Edited Michael Brady and Richard P., MIT Press, Cambridge, MA, USA, (1984), pp. 841-861.

[4] F. Khorrami and U. Ozguner, "Perturbation methods in control of flexible link manipulators", Proceedings of the IEEE International Conference on Robotics and Automation, Philadelphia, PA, USA, (1988), pp. 310-315.

[5] Y. Sakawa, F. Matsuno and S. Fukushima, "Modeling and feedback control of a flexible arm", Journal of Robotic Systems, vol. 2, no 4, (1985), pp. 453-472.

[6] F.Y. Wang, J.T. Wen, "Nonlinear dynamical model and control for a flexible beam", CIRSSE Report No. 75, Rensselaer Polytechnic Institute, Tory, NY, (1990).

[7] F.Y. Wang, "Optimum design of vibrating cantilevers: A classical problem revisited", Journal of Optimization Theory and Applications, vol. 84, no. 3, (1995), pp. 635-652.

[8] S.E. Talole, J.P. Kolhe and S.B. Phadke, "Extended-state-observer-based control of flexible-joint system with experimental validation", IEEE Transactions on Industrial Electronics, vol. 57, no. 4, (2010), pp. $1411-1419$.

[9] M. Mosayebi, M. Ghayour and M.J. Sadigh, “A nonlinear high gain observer based input-output control of flexible link manipulator", Mechanics Research Communications, vol. 45, (2012), pp. 34-41.

[10] S.F. Atashzar, H.A. Talebi, F. Towhidkhah and M. Shahbazi, "Tracking control of flexible-link manipulators based on environmental force disturbance observer", Proceedings of the 49th IEEE Conference on Decision and Control (CDC), Atlanta, GA, (2010), pp. 3584-3589.

[11] S. Kurode and M. Merchant, "Observer based control of flexible link manipulator using discrete sliding modes", Proceedings of the IEEE International Conference on Control Applications (CCA), Hyderabad, India, (2013), pp. 276-281. 
[12] H. Yang, J. Liu and X. Lan, “Observer design for a flexible-link manipulator with PDE model”, Journal of Sound and Vibration, vol. 341, no. 14, (2015), pp. 237-245.

[13] T. Jiang, J. Liu and W. He, "Boundary control for a flexible manipulator based on infinite dimensional disturbance observer", Journal of Sound and Vibration, vol. 348, no. 21, (2015), pp. 1-14.

[14] S. Timoshenko and G.H. MacCullough, "Elements of Strength of Materials", D. Van Nostrand Reinhold, New York, (1964).

[15] M. Takegaki and S. Arimoto, "A new feedback method for dynamic control of manipulators", Journal of Dynamic System, Measurement and Control, vol. 103, no. 2, (1981), pp. 119-125.

[16] Clive L. Dym, Irving and H. Shames, "Solid Mechanics A Variational Approach", Springer, New York, (2013).

[17] Y. Gao, F.Y. Wang and Z.Q. Zhao, "Flexible manipulators: Modeling, Analysis and Optimum Design", first ed., Academic Press, UK, (2012).

[18] M. Hassan, R. Dubay, C. Li and R. Wang, "Active vibration control of a flexible one-link manipulator using a multivariable predictive controller", Mechatronics, vol. 17, no. 6, (2007), pp. 311-323.

[19] M. Zeitz, "The extended Luenberger observer for nonlinear systems", System and Control Letters, vol. 9, no. 2 , (1987), pp. 149-156.

[20] D. Simon, "Optimal State Estimation: Kalman, H Infinity, and Nonlinear Approaches", John Wiley \& Sons, Inc., (2006). 